\title{
A systematic review of gyrase mutations associated with fluoroquinolone-resistant Mycobacterium tuberculosis and a proposed gyrase numbering system
}

\author{
Fernanda Maruri ${ }^{1}$, Timothy R. Sterling ${ }^{1,2 *}$, Anne W. Kaiga ${ }^{3}$, Amondrea Blackman ${ }^{1}$, Yuri F. van der Heijden ${ }^{1}$, \\ Claudine Mayer ${ }^{4-6}$, Emmanuelle Cambau ${ }^{6-8}$ and Alexandra Aubry7,9,10
}

\begin{abstract}
${ }^{1}$ Division of Infectious Diseases, Department of Medicine, Vanderbilt University School of Medicine, Nashville, TN, USA; ${ }^{2}$ Center for Health Services Research, Department of Medicine, Vanderbilt University School of Medicine, Nashville, TN, USA; ${ }^{3}$ Department of Epidemiology, Vanderbilt University Medical Center, Nashville, TN, USA; ${ }^{4}$ Unité de Dynamique Structurale des Macromolécules, Département de Biologie Structurale et Chimie, Institut Pasteur, Paris, France; ${ }^{5}$ URA 2185, Centre National de la Recherche Scientifique (CNRS), Paris, France; ${ }^{6}$ Université Paris Diderot-Paris 07, EA3964, Paris, France; ${ }^{7}$ Centre National de Référence des Mycobactéries et de la Résistance des Mycobactéries aux Antituberculeux, F-75013 Paris, France; ${ }^{8}$ Assistance Publique Hôpitaux de Paris, Groupe Hospitalier Saint LouisLariboisière-Fernand Widal, Paris, France; ${ }^{9}$ Pierre et Marie Curie Université Paris 06, EA1541, Bactériologie-Hygiène, Paris, France;

${ }^{10}$ Assistance Publique Hôpitaux de Paris, Groupe Hospitalier Pitié-Salpêtrière, Paris, France
\end{abstract}

*Corresponding author. Tel: +1-615-322-2035; Fax: +1-615-343-6160; E-mail: timothy.sterling@vanderbilt.edu

Received 6 September 2011; returned 17 October 2011; revised 28 November 2011; accepted 7 December 2011

Fluoroquinolone resistance in Mycobacterium tuberculosis has become increasingly important. A review of mutations in DNA gyrase, the fluoroquinolone target, is needed to improve the molecular detection of resistance. We performed a systematic review of studies reporting mutations in DNA gyrase genes in clinical M. tuberculosis isolates. From 42 studies that met inclusion criteria, 1220 fluoroquinolone-resistant M. tuberculosis isolates underwent sequencing of the quinolone resistance-determining region (QRDR) of gyrA; 780 (64\%) had mutations. The QRDR of gyrB was sequenced in 534 resistant isolates; 17 (3\%) had mutations. Mutations at gyrA codons 90, 91 or 94 were present in 654/1220 (54\%) resistant isolates. Four different GyrB numbering systems were reported, resulting in mutation location discrepancies. We propose a consensus numbering system. Most fluoroquinolone-resistant M. tuberculosis isolates had mutations in DNA gyrase, but a substantial proportion did not. The proposed consensus numbering system can improve molecular detection of resistance and identification of novel mutations.

Keywords: gyrA, gyrB, QRDRs, M. tuberculosis

\section{Introduction}

Fluoroquinolones play an increasingly important role in the treatment of tuberculosis (TB). They are used to treat multidrug-resistant tuberculosis (MDR-TB; defined as resistance to at least isoniazid and rifampicin) and are also recommended to treat drug-susceptible TB in patients with intolerance to first-line antibiotics. ${ }^{1}$ Fluoroquinolones kill bacteria by altering DNA gyrase and DNA topoisomerase IV. Since Mycobacterium tuberculosis does not have topoisomerase IV, fluoroquinolones target DNA gyrase in M. tuberculosis. ${ }^{2}$ DNA gyrase consists of two $A$ and two $B$ subunits encoded by the gyrA and gyrB genes, respectively. ${ }^{3}$ The quinolone resistance-determining region (QRDR) is comprised of conserved areas within gyrA and gyrB in which mutations conferring fluoroquinolone resistance have been reported in most bacterial species, including M. tuberculosis. ${ }^{3-5}$
There are several fluoroquinolone resistance mechanisms in bacteria. Although the primary fluoroquinolone resistance mechanism in M. tuberculosis is related to mutations in the QRDR of DNA gyrase genes, ${ }^{6-12}$ fluoroquinolone resistance can also be conferred by increased fluoroquinolone efflux or DNA mimicry. ${ }^{13-15}$ However, the latter two mechanisms have been described only in laboratory strains, not clinical M. tuberculosis isolates. In many studies, more than $90 \%$ of fluoroquinolone-resistant M. tuberculosis strains have mutations in the QRDR of gyrA or gyrB, ${ }^{16-27}$ so we focused our review on mutations in DNA gyrase.

Conventional phenotypic $M$. tuberculosis drug susceptibility testing (DST) methods for fluoroquinolones require several weeks to complete and are not standardized. Therefore, molecular diagnostic tests for rapid detection of fluoroquinolone resistance at the point-of-care are urgently needed. In addition to fluoroquinolone mono-resistant $M$. tuberculosis isolates, such tests could be used to rapidly identify extensively

(C) The Author 2012. Published by Oxford University Press on behalf of the British Society for Antimicrobial Chemotherapy. All rights reserved. For Permissions, please e-mail: journals.permissions@oup.com 
drug-resistant tuberculosis (XDR-TB; defined as resistance to at least isoniazid and rifampicin plus a fluoroquinolone and a second-line injectable agent such as capreomycin, amikacin and kanamycin). ${ }^{28-30} \mathrm{~A}$ line probe assay has been developed to detect fluoroquinolone-resistance in $M$. tuberculosis, but its sensitivity ranges from $75.6 \%$ to $89.5 \%$ when compared with conventional phenotypic susceptibility methods, ${ }^{19,31,32}$ and $87 \%$ to $100 \%$ when compared with DNA sequencing. ${ }^{31,33}$

The purpose of this review was to characterize all DNA gyrase gene mutations described in M. tuberculosis clinical strains and to distinguish those associated with fluoroquinolone resistance and those reported in fluoroquinolone-susceptible strains. During the review process we found four different numbering systems used for the GyrB subunit, resulting in discrepancies regarding the location of resistance mutations. Some authors used the Escherichia coli numbering system; others used one of three M. tuberculosis numbering systems, which differ according to the position of the start codon. 2,3,34 Most authors did not specify the numbering system used. Even the comprehensive compilation of mutations in the online database http://www.tbdreamdb.com, ${ }^{35}$ which focuses on the QRDR and uses one consistent numbering system, classifies some mutations as inside the QRDR of GyrB when they are indeed outside the QRDR. The different numbering systems make it difficult to confirm previously described mutations and clearly identify novel mutations. We therefore sought to clarify the location of these mutations.

To our knowledge this is the most extensive review to date of mutations described in the gyrA and gyrB genes of fluoroquinolone-resistant and -susceptible strains of M. tuberculosis. It is also the first work to propose a standard numbering system of $M$. tuberculosis GyrA and GyrB, which will allow for accurate comparison of resistance mutations by laboratories around the world.

\section{Methods}

\section{Definitions}

Mutations were defined as nucleotide base-pair changes that resulted in substitutions in amino acids (i.e. non-synonymous), regardless of whether the mutation was reported in a fluoroquinolone-resistant or -susceptible M. tuberculosis isolate. Of those mutations reported in fluoroquinolone-resistant isolates, we distinguished between mutations documented to confer fluoroquinolone resistance by biochemical or genetic experiments and those without such evidence. ${ }^{18,36}$ Polymorphisms were defined as non-synonymous nucleotide base-pair changes known to not be associated with or confer fluoroquinolone resistance. Base-pair changes that did not result in a change in amino acid (i.e. synonymous) were not included in this review. The three-letter abbreviation nomenclature was used to represent amino acids. Substitutions were noted as follows: Xxx\#\#Yyy, where Xxx represents the wild-type amino acid, \#\# the codon number and Yyy the substituted amino acid.

\section{Search strategy}

A computerized search identified peer-reviewed primary research studies reporting fluoroquinolone-resistant or fluoroquinolone-susceptible isolates of $M$. tuberculosis in which mutations in DNA gyrase genes were identified. The search was limited to studies in English published between 1 January 1990 and 30 June 2010. Figure S1 (available as Supplementary data at JAC Online) illustrates the study selection methodology. Full text articles were screened using the Medical Literature Analysis and Retrieval
System Online (MEDLINE) using the keywords 'fluoroquinolone resistance', 'M. tuberculosis', 'gyrA', 'gyrB', 'DNA gyrase', 'mutations', 'drug resistance', 'fluoroquinolone susceptibility', 'second-line drug resistance', 'quinolone resistant' and 'ofloxacin resistance' in different combinations.

The inclusion criteria consisted of (i) publications in which genotypic susceptibility methods were compared with a solid or liquid-based phenotypic resistance reference standard and (ii) DNA gyrase gene mutations were identified in M. tuberculosis isolates obtained from human clinical specimens.

Papers were excluded if they were reviews, letters, duplicates or if the title indicated that the study was not relevant to our study. The online database http://www.tbdreamdb.com ${ }^{35}$ was excluded because it is a compilation of mutations previously reported in the literature rather than a primary source document. Abstracts of the remaining papers were reviewed and studies with irrelevant content were excluded. If the abstract did not provide enough information to include or exclude the article, the entire article was reviewed. Articles were also excluded if they lacked data on amino acid changes or phenotypic susceptibility testing. The bibliographies of the included publications were reviewed, and additional articles not previously identified were added as appropriate.

\section{Data acquisition}

Data abstracted from journal articles that met the inclusion criteria were organized in three ways: (i) all mutations reported in gyrA, (ii) all mutations reported in gyrB and (iii) all combinations of mutations (in gyrA and/or gyrB) reported in a single M. tuberculosis isolate. Although substitutions Glu21Gln, Ser95Thr and Gly668Asp in GyrA result in amino acid changes, they were omitted from the summary tables because they are common polymorphisms that do not correlate with drug resistance. ${ }^{3,37}$

When more than one mutation was observed in one strain (double or triple mutation), we noted two scenarios: (i) either mutation was observed as a single mutation elsewhere or (ii) the mutations were never observed independent of one another. In both scenarios, the mutations were listed as single mutations and as multiple mutations. Mutations that were never observed independently of one another are noted in the tables. This process was designed to capture every mutation without undermining the potential effect that combinations of mutations may have on fluoroquinolone resistance.

For this review, all of the substitutions in GyrB were standardized to the re-annotated genome numbering system of $M$. tuberculosis GyrB, where the QRDR of $M$. tuberculosis GyrB ranges from codon 461 to 499. ${ }^{34}$ Regarding the QRDR of GyrA, some publications used the E. coli numbering system to describe substitution location. ${ }^{38-41}$ In this systematic review, all substitution locations in GyrA were standardized to the genome $M$. tuberculosis numbering system, in which the QRDR of GyrA ranges from codon 74 to $113 .^{3}$

We reported the number of clinical isolates tested, the region sequenced (entire gyrA or gyrB genes or only the QRDRs of gyrA or gyrB), along with the genotypic and phenotypic susceptibility methods used to determine fluoroquinolone resistance for each study. The number of isolates containing a specific mutation was determined and the fluoroquinolone MIC associated with this mutation was included if available. Fluoroquinolone activity (measured as $50 \%$ inhibitory concentration) against $M$. tuberculosis with specific DNA gyrase mutants was also reviewed.

\section{Quality control}

Two authors (F. M. and A. W. K.) independently reviewed and abstracted the data. The data were reviewed for accuracy and compared with particular attention to the numbering systems used. Two additional authors (Y. F. van der H. and A. A.) adjudicated differences between the authors and reviewed the data for accuracy. 


\section{Results}

\section{Numbering systems for M. tuberculosis GyrA and GyrB subunits}

GyrA

The first studies investigating the molecular basis of fluoroquinolone resistance in $M$. tuberculosis were based on E. coli ${ }^{39}$ or $M$. tuberculosis gyrA ${ }^{3}$ gene sequences. Since the GyrA QRDR is located at the N-terminal part of the GyrA subunit and the M. tuberculosis GyrA start codon is seven amino acids before the E. coli GyrA (Figure S2, available as Supplementary data at JAC Online), the amino acids at positions 88, 90, 91 and 94 in the M. tuberculosis numbering system correspond to the amino acids at positions 81,83 , 84 and 87 in the E. coli numbering system, respectively. When these differences were accounted for, there were no discrepancies in the location of substitutions reported in the GyrA QRDR in any of the studies reviewed.

\section{GyrB}

In contrast, four distinct numbering systems have been used to report substitutions in the GyrB QRDR. As a result, in several instances amino acid changes occurring at what was actually the same position were reported as occurring at different positions. For example, substitution Asp472Ala ${ }^{33}$ (2000; Table 1) is the same as substitution Asp500 Ala ${ }^{42}$ (1998; Table 1), but the authors used different numbering systems. Codons 472 and 500 both correspond to position 426 in the E. coli numbering system (Table 1 and Figure S3, available as Supplementary data at JAC Online). Similarly, substitution Asn533Thr ${ }^{26}$ (1994; Table 1) is the same as Asn538Thr ${ }^{42}$ (1998; Table 1); both correspond to codon 464 in the E. coli numbering system. Additionally, discrepancies in the numbering system resulted in differences in classifying mutations as being inside or outside of the QRDR of GyrB. For example, mutations at codons 500 and 501 in the proposed numbering system (2002; Table 1) were designated as inside the QRDR in three studies, but are definitely located outside the QRDR of GyrB. ${ }^{33,42,43}$

Most discrepancies arose from a lack of consensus regarding the gyrB start codon. The first sequence of $M$. tuberculosis gyr $B$ (published in 1994) described the GyrB QRDR extending from codon 495 to $533^{3}$ (see gi|1107468/gb|AAA83016.1| in Figure S3). This numbering system was based on the GyrB E. coli sequence. This numbering system was used for the first studies reporting fluoroquinolone resistance in M. tuberculosis. ${ }^{36}$ When the entire genome of $M$. tuberculosis was published in 1998, the start codon of gyrB was 28 codons upstream of the codon that had been used earlier. Due to the absence of some amino acids in E. coli that are present in M. tuberculosis, the

Table 1. Comparison of the three GyrB numbering systems in M. tuberculosis described in the literature, the corresponding region in E. coli and the proposed consensus numbering system

M. tuberculosis GyrB sequence (year of publication)

\begin{tabular}{|c|c|c|c|c|c|}
\hline \multirow{2}{*}{ Substitution observed } & \multirow[b]{2}{*}{ E. coli ${ }^{a}$} & & & & \\
\hline & & $1994^{\mathrm{b}}$ & $1998^{c}$ & $2000^{d}$ & $2002^{\mathrm{e}}$ proposed numbering system \\
\hline Arg $\rightarrow$ Cys & 411 & 480 & 485 & 457 & 446 \\
\hline Ser $\rightarrow$ Phe & 412 & 481 & 486 & 458 & 447 \\
\hline Asp $\rightarrow$ Ala & 426 & 495 & 500 & 472 & 461 \\
\hline Asp $\rightarrow$ Asn & 426 & 495 & 500 & 472 & 461 \\
\hline Asp $\rightarrow$ His & 426 & 495 & 500 & 472 & 461 \\
\hline Gly $\rightarrow$ Ala & 435 & 504 & 509 & 481 & 470 \\
\hline Asp $\rightarrow$ Ala & 459 & 528 & 533 & 505 & 494 \\
\hline Asn $\rightarrow$ Asp & 464 & 533 & 538 & 510 & 499 \\
\hline Asn $\rightarrow$ Lys & 464 & 533 & 538 & 510 & 499 \\
\hline Asn $\rightarrow$ Thr & 464 & 533 & 538 & 510 & 499 \\
\hline Thr $\rightarrow$ Asn & 465 & 534 & 539 & 511 & 500 \\
\hline Thr $\rightarrow$ Pro & 465 & 534 & 539 & 511 & 500 \\
\hline $\mathrm{Glu} \rightarrow \mathrm{Asp}$ & 466 & 535 & 540 & 512 & 501 \\
\hline $\mathrm{Glu} \rightarrow$ Val & 466 & 535 & 540 & 512 & 501 \\
\hline $\mathrm{Ala} \rightarrow \mathrm{Thr}$ & 469 & 538 & 543 & 515 & 504 \\
\hline $\mathrm{Ala} \rightarrow \mathrm{Val}$ & 469 & 538 & 543 & 515 & 504 \\
\hline $\mathrm{Gln} \rightarrow \mathrm{His}$ & 503 & 572 & 577 & 549 & 538 \\
\hline Deletion & $\Delta 609+610$ & $\Delta 678+679$ & $\Delta 683+684$ & ND & $\Delta 644+645$ \\
\hline
\end{tabular}

ND, not determined.

The QRDR of GyrB is enclosed in the bold box and ranges from codon 461 to codon 499 in the proposed M. tuberculosis GyrB sequence. ${ }^{34}$

aPOAES6.

${ }^{\mathrm{b}} \mathrm{AAA} 83016.1 .^{3}$

'CAB02426.1?

${ }^{\mathrm{d}}$ Zhou et al. $^{44}$

eP0C5C5|1-675. ${ }^{34}$ 
first codon of the GyrB QRDR was five codons upstream of the codon that had been used earlier; the QRDR therefore ranged from amino acid 500 to $538^{2}$ (see gi|1552558|emb|CAB02426.1 in Figure S3). Of the studies reviewed, most authors used the 500-538 numbering system to identify mutation location. However, the first gyrB mutations described in clinical $M$. tuberculosis strains were identified using yet another numbering system, which did not correspond to any of the previously published sequences. The publications using this numbering system did not provide references to justify this numbering sytem. $7,18,21,33,43-45$ In 2002, a re-annotation of the M. tuberculosis genome proposed a new start codon for gyrB. ${ }^{34}$ This numbering system is closer to the $E$. coli numbering system, thus shifting the QRDR to residues $461-499^{34}$ (see POC5C5|1-675 in Figure S3). This latter proposed numbering system appears to be the most accurate. Despite the absence of experiments defining the gyrB start codon, the alignment of M. tuberculosis GyrB sequence and 50 bacterial species (other than $M$. tuberculosis) from five phyla whose sequences were obtained from the SWISS-PROT/TrEMBL (http://expasy.org/sprot) and NCBI (http://www.ncbi.nlm.nih.gov) databases demonstrated that the start codon chosen by Camus et $a .^{34}$ was the most accurate (C. Mayer, personal data). This is the numbering system that we propose future studies use as the reference regarding fluoroquinolone resistance in $M$. tuberculosis.

Table 1 compares the three M. tuberculosis GyrB numbering systems used in the literature as well as the E. coli GyrB numbering system. The consensus numbering system that we propose is also included.

\section{Findings}

Forty-two publications met the inclusion criteria. From these studies, a total of $2482 \mathrm{M}$. tuberculosis isolates were assessed for genotypic mutations: 1220 (49\%) were phenotypically fluoroquinolone resistant and 1262 (51\%) were fluoroquinolone susceptible. The phenotypic and genotypic resistance methods used and the regions of $M$. tuberculosis DNA sequenced are provided in Table 2. Twenty-seven studies sequenced the QRDR of gyrA, 13 sequenced the QRDR of both gyrA and gyrB, 1 sequenced the entire gyrA and 1 sequenced the entire gyrA and gyrB genes (Table 2).

There were gyrA or gyrB mutations identified in $806 / 1220$ (66\%) phenotypically resistant isolates and 19/1262 (2\%) phenotypically susceptible isolates. Specific mutations identified in each gyrA and gyrB gene are described in the following sections.

Among the 2482 isolates tested, 44 distinct mutations were identified. Of the 44 mutations, 26 (59\%) were in GyrA and 18 $(41 \%)$ in GyrB; they occurred at 24 different codons. Of the 44 mutations, 34 (77\%) occurred in phenotypically resistant M. tuberculosis isolates, 5 (11\%) in phenotypically susceptible isolates and $5(11 \%)$ in both resistant and susceptible isolates. These findings are discussed in greater detail below and in Tables 3-5.

\section{Mutations in gyrA}

Of the 26 GyrA mutations, 21 (81\%) were inside the QRDR and 5 (19\%) were outside the QRDR, including 1 (4\%) that was in the putative promoter (Table 3). Of the 1220 fluoroquinolone-resistant isolates sequenced, 780 (64\%) had mutations inside the QRDR of GyrA and $6(0.5 \%)$ had mutations outside of the QRDR of GyrA (Table 3). Substitutions at codon 94 were the most prevalent; they were reported in $37 \%$ of the fluoroquinolone-resistant strains. Various substitutions were reported at codon 94 in GyrA: aspartic acid (Asp) was replaced by alanine (Ala), asparagine (Asn), glycine (Gly), histidine (His), phenylalanine (Phe), tyrosine (Tyr) or valine (Val). The Asp94Gly substitution in GyrA occurred most frequently; it was reported in 234 (19\%) resistant isolates. Overall, mutations at codons 90,91 or 94 were reported in 654 (54\%) of the 1220 fluoroquinolone-resistant isolates, with substitutions at codons 90 and 91 occurring in 13\% and $4 \%$ of the isolates, respectively.

Of the 1262 fluoroquinolone-susceptible isolates studied for GyrA, 14 (1\%) had mutations inside the QRDR of GyrA and 2 had a substitution in the putative promoter of GyrA ${ }^{26}$ (Table 3).

\section{Mutations in gyrB}

Of the 18 GyrB mutations, 8 (44\%) were inside the QRDR, 9 (50\%) were outside the QRDR and 1 (6\%) was a deletion also outside the QRDR (Table 4). Although all 42 studies sequenced the GyrA QRDR, only 14 studies sequenced GyrB (mostly the QRDR) (Table 2). Of the 534 resistant isolates studied for gyrB, 17 (3\%) harboured mutations in the QRDR and 13 (2\%) harboured mutations outside of the QRDR. Ten (2\%) isolates had a substitution at codon 499; Asn499Asp (in the proposed numbering system, 2002; Table 1) was reported in $4(0.7 \%)$ of the 10 resistant isolates. In one case, deletion of codons 644 and 645 in GyrB was reported in a fluoroquinolone-resistant strain. ${ }^{26}$ Among the 377 susceptible strains, there was one substitution reported in GyrB (Ser447Phe in the proposed numbering system; Table 1) outside the QRDR. Codon mutations and associated MICs are presented in Table 6.

\section{Multiple mutations and heteroresistance}

Several studies reported multiple mutations in gyrA, gyrB or in both gyrA and gyrB (Table 5), with up to five mutations in the same strain. ${ }^{42}$ Double mutations in gyra have often been described as two mutations in the same gyrA allele and have been associated with high-level resistance. ${ }^{36}$ They may result from a two-step selection of fluoroquinolone-resistant mutants. ${ }^{18}$ The combination of Ala90Val with Asp94Gly is frequently associated with high-level resistance and was reported in 34 (3\%) of the fluoroquinolone-resistant isolates. ${ }^{19,21,33,42,46,47}$ The combination of Thr80Ala with Ala90Gly, which was demonstrated to confer fluoroquinolone susceptibility $^{18}$ was the most frequent double mutation reported among fluoroquinolone-susceptible isolates [6 (0.5\%) isolates] (Tables 5-7).

In several studies, sequencing showed heterogeneous peaks in up to $31.2 \%$ of the isolates. ${ }^{19,42,48}$ Such heteroresistance refers to the concomitant presence of susceptible and resistant bacilli in the same clinical specimen or the presence of several clones, each harbouring a distinct mutation. ${ }^{49}$ It probably results from a strain splitting into two or more bacterial clones or less likely from infection by two different strains. ${ }^{50}$

Mutations that conferred fluoroquinolone resistance (rather than simply being present in fluoroquinolone-resistant 
Table 2. Clinical M. tuberculosis isolates studied: fluoroquinolone susceptibility and molecular detection methods used in each primary study included in the review

\begin{tabular}{|c|c|c|c|c|c|c|c|}
\hline Type of collection & $\begin{array}{l}\text { No. of } \\
\text { FQR }\end{array}$ & $\begin{array}{l}\text { No. of } \\
\text { FQS }\end{array}$ & $\begin{array}{c}\text { Susceptibility method (OFX } \\
\text { breakpoints) }\end{array}$ & $\begin{array}{c}\text { Molecular detection } \\
\text { method }\end{array}$ & $\begin{array}{l}\text { DNA region } \\
\text { studied }^{a}\end{array}$ & $\begin{array}{c}\text { Percentage FQR with } \\
\text { mutations }\end{array}$ & $\begin{array}{l}\text { Study } \\
\text { reference }\end{array}$ \\
\hline ND & 4 & 0 & PMS on $7 \mathrm{H} 11$ (2 mg/L) & PCR seq & QRDR_A & 100 & 25 \\
\hline ND & 8 & 1 & ACM on LJ (2 mg/L) & PCR seq & QRDR_A & 100 & 27 \\
\hline ND & 87 & 0 & ACM on LJ & PCR seq & QRDR_A & 100 & 46 \\
\hline HIV-infected TB cases & 1 & 0 & $\begin{array}{l}\text { MIC in liquid media } \\
\text { (7H12 + Bactec) }\end{array}$ & PCR seq & QRDR_A & 100 & 60 \\
\hline MDR & 52 & 55 & ACM on LJ (2 mg/L) & microchips & QRDR_A & 98 & 17 \\
\hline ND & 71 & 47 & ACM on LJ (8 mg/L) & PCR seq & QRDR_A & 97.2 & 24 \\
\hline XDR & 26 & 0 & PML (MGIT 960+Bactec 460) & PCR seq & QRDR_A & 96.2 & 22 \\
\hline MDR & 35 & 108 & $\begin{array}{l}\text { ACM on LJ }(2.4 \mathrm{mg} / \mathrm{L})+\mathrm{PML} \\
\quad(\mathrm{MGIT} 960)\end{array}$ & PCR seq & QRDR_A & 94.3 & 20 \\
\hline MDR & 11 & 43 & PMS on $7 H 11$ (2 mg/L) & MAS-PCR & QRDR_A & 91 & 53 \\
\hline $\begin{array}{l}\text { Fluoroquinolone, AMK, CAP and/or } \\
\text { EMB resistance }\end{array}$ & 32 & 74 & $\begin{array}{l}\text { PML (MGIT 960)+PMS on LJ } \\
\quad(2 \mathrm{mg} / \mathrm{L})\end{array}$ & PCR seq + MTBDRsl & QRDR_A & 90.6 & 19 \\
\hline ND & 19 & 9 & MIC on $7 \mathrm{H} 11$ (2 mg/L) & PCR seq + line probe assay & QRDR_A & 89.5 & 31 \\
\hline ND & 30 & 0 & PMS on LJ (2 mg/L) & PCR seq & QRDR_A & 86.7 & 61 \\
\hline XDR & 13 & 0 & MIC in solid medium (5 mg/L) & PCR seq & QRDR_A & 85 & 62 \\
\hline ND & 13 & 6 & $\begin{array}{l}\text { growth inhibition in liquid } \\
\text { culture (ID50) }\end{array}$ & PCR seq & QRDR_A & 84.6 & 63 \\
\hline Drug resistant & 71 & 179 & $\mathrm{ACM}(4.8 \mathrm{mg} / \mathrm{L})$ & PCR-SSCP/MPAC + PCR seq & QRDR_A & 78 & 54 \\
\hline ND & 42 & 40 & PMS on LJ ( 2 mg/L) & LNA-PCR & QRDR_A & 76.2 & 48 \\
\hline ND & 10 & 92 & disc proportion method & pyrosequencing & QRDR_A & 70 & 55 \\
\hline ND & 110 & 0 & MIC (5 mg/L) & PCR seq, DHPLC & QRDR_A & 67.5 & 47 \\
\hline ND & 12 & 0 & PMS (2 mg/L) & PCR seq & QRDR_A & 66.7 & 12 \\
\hline ND & 55 & 83 & ACM on LJ ( 2 mg/L) & PCR seq & QRDR_A & 58 & 6 \\
\hline ND & 4 & 0 & $\begin{array}{l}\text { PMS on } \mathrm{LJ}+\text { MIC in solid } \\
\text { medium }(2 \mathrm{mg} / \mathrm{L})\end{array}$ & PCR seq & QRDR_A & 50 & 11 \\
\hline ND & 1 & 0 & PMS on LJ (2 mg/L) & PCR seq & QRDR_A & ND & 39 \\
\hline ND & 0 & 16 & $\mathrm{PML}$ in $7 \mathrm{H9}$ & PCR seq & QRDR_A & ND & 40 \\
\hline ND & 2 & 0 & MIC on LJ & PCR seq & QRDR_A & ND & 64 \\
\hline ND & 2 & 0 & MIC on $7 \mathrm{H} 10$ (2 mg/L) & PCR seq & QRDR_A & ND & 38 \\
\hline ND & 3 & 3 & MIC (2 mg/L) & $\begin{array}{l}\text { non-radioactive PCR-SSCP, } \\
\text { PCR seq }\end{array}$ & QRDR_A & ND & 41 \\
\hline ND & 16 & 0 & PML, Bactec 460 & PCR-SSCP, PCR seq & QRDR_A & ND & 65 \\
\hline ND & 15 & 39 & PML (Bactec) (2 mg/L) & $\begin{array}{l}\text { non-radioactive PCR-SSCP, } \\
\text { PCR seq }\end{array}$ & QRDR_AB & 100 & 3 \\
\hline ND & 4 & 4 & MIC in 7H11 (2 mg/L) & PCR seq & QRDR_AB & 100 & 18 \\
\hline ND & 24 & 28 & PMS on LJ (2 mg/L) & PCR seq+MTBDRsl & QRDR_AB & 100 & 33 \\
\hline MDR & 26 & 49 & PMS (2 mg/L) & PCR seq & QRDR_AB & 96 & 16 \\
\hline FQR and MDR & 48 & 23 & ACM (2 mg/L) & PCR seq & QRDR_AB & 91.7 & 21 \\
\hline Consecutive FQR & 109 & 0 & PMS on LJ (2 mg/L) & PCR seq & QRDR_AB & 82.6 & 42 \\
\hline ND & 60 & 2 & ACM on $7 \mathrm{H} 11$ & PCR seq & QRDR_AB & 73.3 & 43 \\
\hline
\end{tabular}




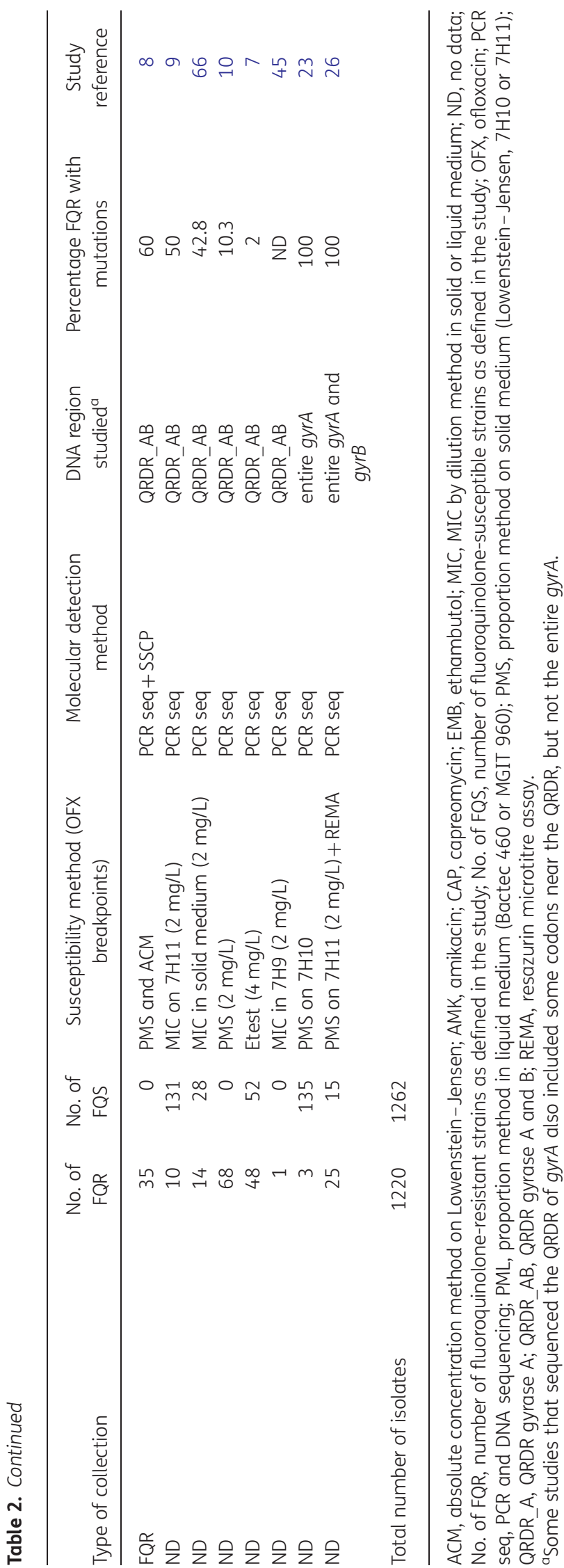

M. tuberculosis isolates) were identified based on biochemical studies demonstrating that the altered DNA gyrase subunit was resistant to fluoroquinolone inhibition. Table 7 lists the mutations that have been demonstrated to confer fluoroquinolone resistance.

\section{Discussion}

Because M. tuberculosis is a slow-growing bacterium, the detection of drug resistance by traditional methods requires 5-12 weeks. Several studies have demonstrated that molecular tests facilitate rapid diagnosis of resistance in $M$. tuberculosis, including MDR-TB and XDR-TB, which can occur 2-3 months faster than with conventional susceptibility testing. ${ }^{51}$ To maximize the sensitivity and specificity of molecular testing for fluoroquinolone resistance in $M$. tuberculosis, it is critical to have comprehensive knowledge of the fluoroquinolone resistance mutations.

Because the primary mechanism of fluoroquinolone resistance in M. tuberculosis occurs via modification of DNA gyrase, it is important to (i) summarize all mutations described in DNA gyrase genes of $M$. tuberculosis and (ii) clarify the role of these mutations in fluoroquinolone resistance.

Our review differs from the compilation of mutations in tbdream, ${ }^{35}$ in that our review includes all mutations reported outside the QRDR of gyrA and gyrB (including heterogeneity at codons), addresses the four different gyrB numbering systems, provides the prevalence of common resistance mutations, notes which mutations have been demonstrated to confer resistance and provides the range of MICs reported for mutations at each codon.

We needed first to clarify the discrepancies of the GyrB numbering system to be able to differentiate novel from previously described mutations. We propose that the GyrB sequence that was re-annotated in $2002^{34}$ should be used as the reference sequence (Table 1 and Figure S3).

Having clarified the numbering system of GyrB, we reviewed all the mutations described in the DNA gyrase genes of clinical M. tuberculosis isolates.

Mutations at GyrA codons 90,91 or 94 were most common, $3,6-8,38,52$ and seen in 654 (54\%) resistant isolates. Although a majority of fluoroquinolone-resistant $M$. tuberculosis isolates had mutations in these codons, a substantial proportion did not. This suggests that the sensitivity of line-probe assays or other molecular diagnostic tests that focus on these codons could have low sensitivity. However, the isolates in this systematic review, although representing all fluoroquinolone-resistant M. tuberculosis isolates reported to date, and encompassing multiple countries of the world (e.g. China, France, India, Russia, the USA, Uzbekistan and Vietnam), may differ from population-based studies.

Based on our review, the most widely used molecular method to detect fluoroquinolone resistance in $M$. tuberculosis was PCR and DNA sequencing (Table 2). Other, more rapid testing methods included multiplex allele-specific PCR (MAS-PCR), ${ }^{53}$ PCR single-stranded conformation polymorphism/multiplex PCR amplimer conformation (PCR-SSCP/MPAC), ${ }^{54}$ pyrosequencing, ${ }^{55}$ denaturing HPLC (DHPLC), ${ }^{47}$ non-radioactive PCR-SSCP, ${ }^{3,8,41}$ locked nucleic acid probe real-time PCR (LNA-PCR) ${ }^{48}$ and 
Table 3. GyrA substitutions reported in fluoroquinolone-resistant and -susceptible clinical M. tuberculosis isolates

\begin{tabular}{|c|c|c|}
\hline \multirow[b]{2}{*}{ Substitution $^{a}$} & \multicolumn{2}{|l|}{ Study reference } \\
\hline & fluoroquinolone-resistant isolates & $\begin{array}{l}\text { fluoroquinolone-susceptible } \\
\text { isolates }\end{array}$ \\
\hline $\begin{array}{l}\text { Putative } \\
\text { promoter }\end{array}$ & none & 26 \\
\hline Pro8Ala & none & 26 \\
\hline Arg68Gly & none & 24 \\
\hline His70Arg & 43 & none \\
\hline Ala74Ser & 46,47 & none \\
\hline Thr80Ala & $18,25,33$ & $26,33,40$ \\
\hline Gly88Ala & $9,33,42,48,64$ & none \\
\hline Gly88Cys & $8,17,21,33,54,60$ & none \\
\hline Asp89Asn & 26,48 & none \\
\hline Ala90Glu & 33 & none \\
\hline Ala90Gly & 33 & $18,26,33$ \\
\hline Ala90Leu & 11 & none \\
\hline Ala90Val & $\begin{array}{l}3,6,8,9,10,12,16,17,18,19,20,21,23,24,26,27,31,33,42,43,46,47,48,53,54,55 \\
\quad 61,62,63,65,66\end{array}$ & 17 \\
\hline Ser91Ala & 43 & none \\
\hline Ser91Pro & $3,6,8,10,16,17,19,20,22,24,27,31,42,46,47,48,54,65$ & none \\
\hline Ile92Met & 11 & none \\
\hline Asp94Ala & $3,6,8,10,12,16,17,19,20,21,22,23,24,25,26,31,33,38,42,43,46,47,48,54,61,62$ & 54 \\
\hline Asp94Asn & $3,6,8,12,16,17,19,21,24,26,27,31,33,38,42,43,46,47,54,62,63$ & none \\
\hline Asp94Gly & $\begin{array}{l}3,6,8,9,10,12,16,17,19,20,21,22,23,24,26,27,31,33,41,42,43,46,47,48,53,54 \\
55,61,62,63,65,66\end{array}$ & none \\
\hline Asp94His & $3,6,8,12,16,17,20,26,33,39,41,42,47,54,62,63$ & none \\
\hline Asp94Phe & 55 & none \\
\hline Asp94Tyr & $3,6,9,12,16,17,19,20,21,26,41,42,46,47,48,54,61,62,63$ & none \\
\hline Asp94Val & 24 & none \\
\hline Pro $102 \mathrm{His}$ & none & 20 \\
\hline Leu109Val & none & 24 \\
\hline Ala126Arg & 20,54 & none \\
\hline
\end{tabular}

The QRDR is enclosed in the bold box and ranges from codon 74 to codon 113.

Substitutions not demonstrated to confer fluoroquinolone resistance in $M$. tuberculosis are in italics. ${ }^{18}$

Substitutions demonstrated to confer fluoroquinolone resistance in M. tuberculosis are in bold. ${ }^{18,64}$

Substitutions not italicized or in bold have not been assessed for conferring resistance.

apolymorphisms Glu21Gln, Ser95Thr and Gly668Asp were not included. ${ }^{37}$

bSubstitutions Ala74Ser and Ala90Gly were never observed independently. ${ }^{18,26,33,46,47}$

GenoType ${ }^{\circledR}$ MTBDRsl (Hain Lifescience) line probe. ${ }^{19,31,33}$ These rapid genotypic assays detect fluoroquinolone resistance in isolated cultures of $M$. tuberculosis, ${ }^{28}$ and less frequently, directly in respiratory specimens. ${ }^{51}$

Knowledge of all mutations that are involved in fluoroquinolone resistance will help in the design of more sensitive and specific rapid molecular tests. We based our assessment of specific mutations that confer fluoroquinolone resistance on biochemical studies demonstrating that the altered DNA gyrase subunit was resistant to fluoroquinolone inhibition (Table 7). Discrepancies between the results of drug susceptibility testing and biochemical studies can exist since clinical isolates may harbour additional mutations or resistance mechanisms. There are several fluoroquinolone resistance mechanisms in bacteria besides mutations in the gyrA and gyrB genes. These include mutations in the parC and parE genes, enhancement of efflux pumps and plasmid-mediated mechanisms such as anr genes encoding pentapeptide repeat proteins, aac (6')-Ib-cr encoding a variant aminoglycoside acetyltransferase and oqxAB and qepA encoding efflux pumps. ${ }^{5}$ For example, biochemical studies on mutant DNA gyrases have demonstrated that the GyrA mutations Thr80Ala and Ala90Gly, and GyrB mutation Ser447Phe do not confer resistance, but these mutations were found in resistant isolates. This may have been because the isolates were misclassified or resistance was conferred by another mechanism (Tables 3, 4 and 7). ${ }^{18,25,33,42}$ On the other hand, it is highly surprising to observe that some strains carrying mutations that have previously been demonstrated to confer fluoroquinolone resistance are susceptible to fluoroquinolones (Tables 3 and 7). ${ }^{17,54}$ Such discrepancies may be due to misclassification of the strain as susceptible, 
Table 4. GyrB substitutions reported in fluoroquinolone-resistant and -susceptible clinical M. tuberculosis isolates

\begin{tabular}{|c|c|c|c|}
\hline \multirow[b]{2}{*}{$\begin{array}{l}\text { Substitution named by } \\
\text { author }^{a}\end{array}$} & \multirow[b]{2}{*}{$\begin{array}{l}\text { Substitution in proposed numbering } \\
\text { system }^{\text {b }}\end{array}$} & \multicolumn{2}{|c|}{ Study reference } \\
\hline & & $\begin{array}{l}\text { fluoroquinolone-resistant } \\
\text { isolates }\end{array}$ & $\begin{array}{l}\text { fluoroquinolone-susceptible } \\
\text { isolates }\end{array}$ \\
\hline Arg485Cys ${ }^{c}$ & Arg446Cys & 16 & none \\
\hline Ser486Phe ${ }^{c}$ & Ser447Phe & 42 & 33 \\
\hline $\begin{array}{l}\text { Asp472Aa }{ }^{\mathrm{d}} \\
\text { Asp500Ala }\end{array}$ & Asp461Ala & 33,42 & none \\
\hline $\begin{array}{l}\text { Asp495Asn } \\
\text { Asp500Asn }\end{array}$ & Asp461Asn & 8,42 & none \\
\hline Asp500His ${ }^{c}$ & Asp461His & 42 & none \\
\hline Gly509Alac & Gly470Ala ${ }^{f}$ & 42 & none \\
\hline Asp505Ala ${ }^{d}$ & Asp494Ala & 7 & none \\
\hline Asn510Asp ${ }^{d}$ & Asn499Asp & $18,33,45,66$ & none \\
\hline Asn538Asp ${ }^{C}$ & & & \\
\hline Asn510 Lys $^{\mathrm{d}}$ & Asn499 Lys $^{f}$ & 21 & none \\
\hline Asn538Thr & Asn499Thr & 26,42 & none \\
\hline Asn533Thr ${ }^{\mathrm{e}}$ & & & \\
\hline Thr511Asn ${ }^{d}$ & Thr500Asn & 43 & none \\
\hline Thr511Pro ${ }^{d}$ & Thr500Pro & 33,42 & none \\
\hline Thr539Proc & & & \\
\hline Glu540Asp c & Glu501Asp & 42 & none \\
\hline Glu540Val $^{c}$ & Glu501Val & 42 & none \\
\hline Ala543Thrc & Ala504Thr & 16,21 & none \\
\hline Ala515Thr $^{\mathrm{d}}$ & & & \\
\hline Ala515Val $^{d}$ & Ala504Val & 21 & none \\
\hline Gln549His ${ }^{d}$ & Gln538His & 21 & none \\
\hline$\Delta 678+679^{e}$ & $\Delta 644+645^{f}$ & 26 & none \\
\hline
\end{tabular}

The QRDR is enclosed in the bold box.

Substitutions not demonstrated to confer fluoroquinolone resistance in M. tuberculosis are in italics. ${ }^{67}$

Substitutions demonstrated to confer fluoroquinolone resistance in M. tuberculosis are in bold. ${ }^{18,64}$

${ }^{a}$ See Table 1 for corresponding M. tuberculosis numbering systems.

bSubstitutions are standardized to POC5C5|1-675, ${ }^{34}$ where the QRDR ranges from codon 461 to codon 499.

${ }^{\mathrm{C} C A B 02426.1 .^{2}}$

d'hou et al. $^{44}$

${ }^{\text {eAAA83016.1. }}{ }^{3}$

fSubstitutions Gly470Ala, Asn499Lys and $\Delta 644+645$ were never observed independently. ${ }^{21,26,42}$

an error in the molecular detection of resistance or the presence of a mixture of strains. For example, in a specimen that harbours mutations leading to GyrA Ala90Val, Asp94Ala and wild-type alleles, results of phenotypic susceptibility testing may have reflected only the wild-type strain.

It is likely that all substitutions observed at codon 94 in GyrA confer fluoroquinolone resistance, even if biochemical tests have not been performed for all substitutions described at this codon. Similarly, it is likely that substitutions at codon 91, reported in fluoroquinolone-resistant strains in 19 studies, are responsible for fluoroquinolone resistance in M. tuberculosis (Tables 3 and 6). However, less frequently described mutations such as His70Arg, Asp89Asn and Ile92Met in GyrA and most GyrB mutations (except Ser447Phe and Asn499Asp previously studied) deserve biochemical studies to determine whether or not they confer fluoroquinolone resistance. It is especially important since it has been shown that some mutations may confer high levels of resistance or hypersusceptibility depending on the amino acid substitution. ${ }^{18}$ Indeed, it has been previously demonstrated that when alanine at codon 90 of GyrA is replaced by valine, the fluoroquinolone affinity of the resulting enzyme decreases compared with the wild-type, resulting in resistance. However, when it is replaced by glycine the fluoroquinolone affinity of the resulting enzyme increases, resulting in susceptibility (Table 7$)^{18}$

Although the majority of the described mutations occur in the QRDR of GyrA and GyrB (Tables 3-5), some substitutions were described outside of the QRDR. Further studies are necessary to characterize the significance and relevance of these mutations, especially in GyrB, where they are seen more frequently. The GyrB QRDR may need to be redefined if it is determined that substitutions at codons 500-504 (in the proposed numbering system, 2002; Table 1) are implicated in fluoroquinolone resistance. 
Table 5. Multiple substitutions in GyrA, GyrB or both GyrA and GyrB reported in fluoroquinolone-resistant and -susceptible clinical M. tuberculosis isolates

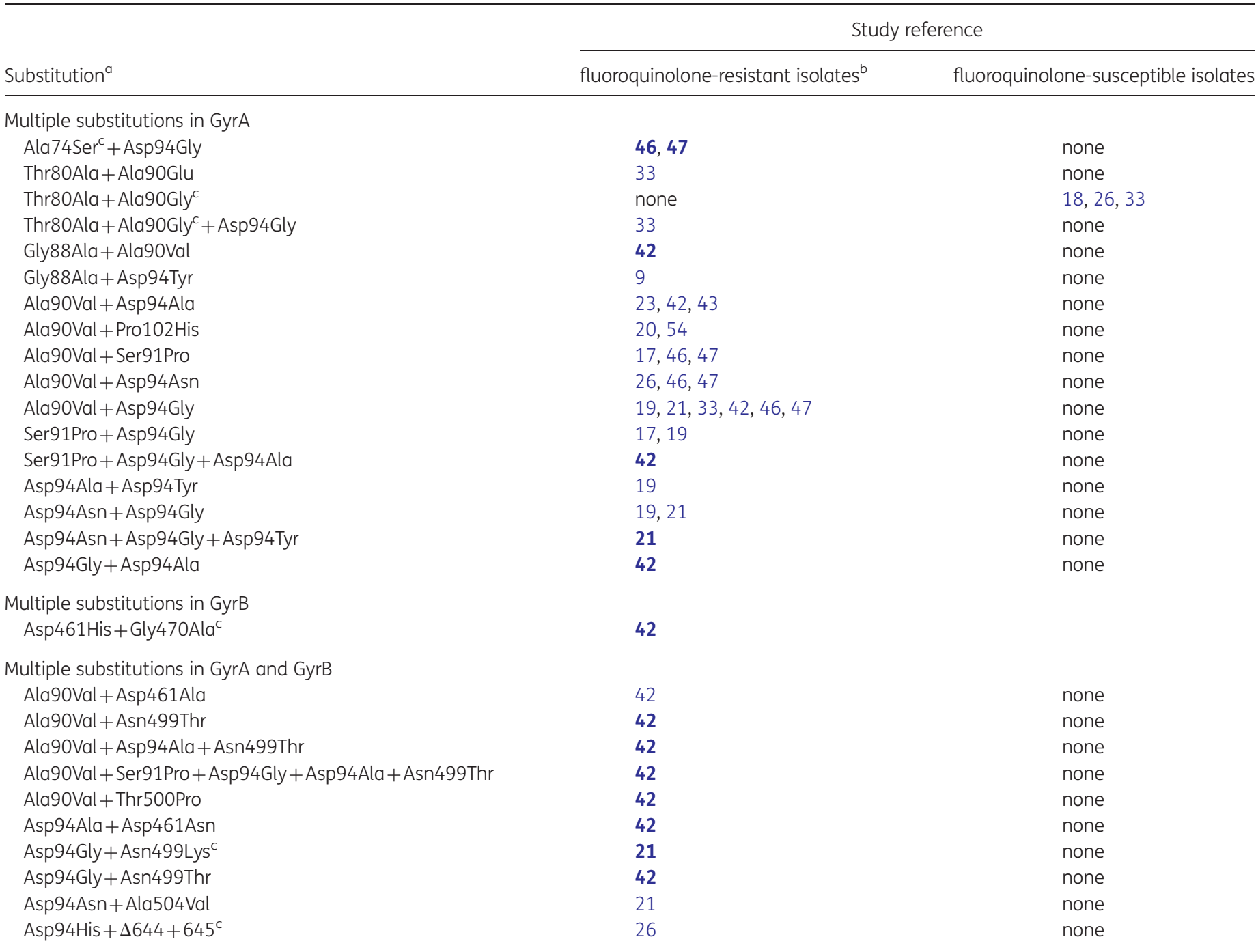

a Substitutions in GyrB are standardized to POC5C5|1-675, ${ }^{34}$ where the QRDR ranges from codon 461 to codon 499 . See Table 1 for corresponding sequences.

beferences where heteropeaks were reported are in bold.

'Substitutions GyrA Ala74Ser and Ala90Gly, and GyrB Gly470Ala, Asn499Lys and $\Delta 644-645$ were never observed independently. ${ }^{18,21,26,33,42,46,47}$

In several studies, gyrA or gyrB mutations were revealed as heteropeaks, which are a combination of either wild-type and mutated alleles, or different mutated alleles. ${ }^{19,21,42,46}$ Heteropeaks in fluoroquinolone-resistant $M$. tuberculosis have been described in detail previously. ${ }^{48}$ In contrast to most bacteria, M. tuberculosis acquisition of drug resistance does not occur as a result of horizontal transfer of resistance-bearing genetic elements. ${ }^{56}$ Selection of drug resistance during treatment of TB results in a mixture of subpopulations of bacilli, some of which bear different genetic mutations that account for the drug resistance, whereas others are drug susceptible. Consequently, the simultaneous presence of drug-resistant and drug-susceptible bacilli, also designated as heteroresistance, may be found in sputum. ${ }^{56}$ These heteroresistant isolates are distinct from isolates with multiple mutations. In fact, fluoroquinolone MICs for isolates with multiple mutations are much higher than those for strains harbouring one mutation, even if presented as a polyclonal population (Table 6). In the presence of anti-TB drugs, drug-resistant bacilli would be expected to outgrow drugsusceptible bacilli over time.

New WHO guidelines include the use of moxifloxacin for the treatment of XDR-TB patients. ${ }^{57}$ Although there is crossresistance within the fluoroquinolone group, moxifloxacin MICs are usually lower than those of other fluoroquinolones (Table 6), ${ }^{18,20,58}$ making it a potentially effective drug to treat ofloxacin-resistant TB. The WHO recommendation is supported by a few clinical observations, ${ }^{59}$ and also by experiments performed in murine models. ${ }^{58}$ 
Table 6. Number of M. tuberculosis isolates with specific codon mutations and the fluoroquinolone MICs for these isolates

\begin{tabular}{|c|c|c|c|c|}
\hline Codon $^{a}$ & $\begin{array}{l}\text { Number of isolates } \\
\text { with this mutation }\end{array}$ & $\begin{array}{l}\text { OFX MIC range } \\
\quad(\mathrm{mg} / \mathrm{L})\end{array}$ & $\begin{array}{l}\text { MXF MIC range } \\
\quad(\mathrm{mg} / \mathrm{L})\end{array}$ & Study reference \\
\hline \multicolumn{5}{|l|}{ Single mutations in gyrA } \\
\hline putative promoter & 2 & 1 & $\leq 0.125$ & 26 \\
\hline 8 & 1 & 0.5 & $\leq 0.125$ & 26 \\
\hline 68 & 1 & $<2$ & & 24 \\
\hline 70 & 2 & 1 & & $43^{b}$ \\
\hline 80 & 7 & $0.2->4$ & $\leq 0.125-1$ & $18,25,26,40^{b}$ \\
\hline $88^{a}$ & 11 & $2-16$ & 2 & $8,17,21,{ }^{c} 33,{ }^{c} 48,{ }^{c} 54,60,{ }^{b} 64$ \\
\hline 89 & 2 & $>2-4$ & 2 & $26,48^{c}$ \\
\hline $90^{a}$ & 167 & $0.5-20$ & $0.25-4$ & $\begin{array}{l}3,,^{b} 6,8,9,10,{ }^{c} 11,{ }^{c} 12,16,17,18,19,{ }^{c} 20,21,23,24,26,27, \\
31,33,42,43,{ }^{b} 46,47,48,53,54,55,61,{ }^{b} 62,63,{ }^{b, c} 65,66^{c}\end{array}$ \\
\hline $91^{a}$ & 44 & $1-64$ & $1-2$ & $\begin{array}{l}3,,^{b} 6,8,10,{ }^{c} 16,17,19,{ }^{c} 20,22,24,27,31,42,43,{ }^{b} 46,47 \\
\quad 48,{ }^{c} 54,65\end{array}$ \\
\hline 92 & 1 & 4 & & $11^{\mathrm{b}}$ \\
\hline $94^{a}$ & 447 & $0.5-64$ & $0.5-8$ & $\begin{array}{l}3,^{b} 6,8,9,10,12,16,17,19,20,21,,^{c} 22,23,24,25,{ }^{c} 26,27 \\
31,33,{ }^{c} 38,39,41,42,43,{ }^{b} 46,47,48,{ }^{c} 53,54,55,61,{ }^{b} 62 \\
63,{ }^{b} 65,{ }^{c} 66^{c}\end{array}$ \\
\hline 102 & 1 & ND & & 20 \\
\hline 109 & 1 & 2 & & 24 \\
\hline 126 & 4 & $4.8-8$ & & 20,54 \\
\hline \multicolumn{5}{|l|}{ Single mutations in gyrB } \\
\hline 446 & 1 & ND & & 16 \\
\hline 447 & 2 & ND & & 33,42 \\
\hline 461 & 3 & $>2-4$ & & $8,33,{ }^{c} 42$ \\
\hline 494 & 1 & $>32$ & & 7 \\
\hline 499 & 5 & $1-8$ & $0.25-4$ & $18,26,33,{ }^{c} 45,66$ \\
\hline 500 & 2 & $1->2$ & & $33,,^{c} 43^{b}$ \\
\hline 501 & 2 & 12 & & 42 \\
\hline $504^{a}$ & 3 & $>2$ & & $16,21^{\mathrm{c}}$ \\
\hline 538 & 1 & $>2$ & & $21^{c}$ \\
\hline \multicolumn{5}{|l|}{ Multiple mutations in gyrA } \\
\hline $74+94$ & 21 & $2-32$ & & 46,47 \\
\hline $80+90$ & 7 & $<0.25->2$ & $<0.12-0.25$ & $18,26,33$ \\
\hline $80+90+94$ & 1 & $>2$ & & $33^{c}$ \\
\hline $88+90$ & 1 & ND & & 42 \\
\hline $88+94$ & 1 & $>2$ & & $9^{c}$ \\
\hline $90+91$ & 11 & $2-20$ & & $17,46,47$ \\
\hline $90+94^{a}$ & 48 & $12-32$ & $>8$ & $19,,^{c} 21,{ }^{c} 23,26,33^{c} 42,{ }^{c} 43,{ }^{b} 46,47$ \\
\hline $90+102$ & 3 & $4-4.8$ & & 20,54 \\
\hline $91+94$ & 2 & $>2$ & & 17,19 \\
\hline $91+94+94$ & 1 & ND & & 42 \\
\hline $94+94^{a}$ & 6 & $>2-12$ & & $19,{ }^{\mathrm{C}} 21,{ }^{\mathrm{C}} 42$ \\
\hline $94+94+94^{a}$ & 1 & $>2$ & & $21^{c}$ \\
\hline \multicolumn{5}{|l|}{ Multiple mutations in gyrB } \\
\hline $461+470^{a}$ & 1 & ND & & 42 \\
\hline \multicolumn{5}{|c|}{ Multiple mutations in gyrA and gyrB } \\
\hline $90+461^{a}$ & 1 & 8 & & 42 \\
\hline $90+499^{a}$ & 1 & ND & & 42 \\
\hline $90+94+499^{a}$ & 1 & ND & & 42 \\
\hline $90+91+94+94+499^{a}$ & 1 & ND & & 42 \\
\hline $90+500$ & 1 & $>12$ & & 42 \\
\hline
\end{tabular}


Table 6. Continued

\begin{tabular}{|c|c|c|c|c|}
\hline $\operatorname{Codon}^{a}$ & $\begin{array}{l}\text { Number of isolates } \\
\text { with this mutation }\end{array}$ & $\begin{array}{l}\text { OFX MIC range } \\
(\mathrm{mg} / \mathrm{L})\end{array}$ & $\begin{array}{l}\text { MXF MIC range } \\
(\mathrm{mg} / \mathrm{L})\end{array}$ & Study reference \\
\hline $94+461^{a}$ & 1 & ND & & 42 \\
\hline $94+499^{a}$ & 2 & $>2$ & & $21,{ }^{\mathrm{C}} 42$ \\
\hline $94+504$ & 1 & $>2$ & & $21^{c}$ \\
\hline $94+\Delta 644+645$ & 1 & 4 & 2 & 26 \\
\hline
\end{tabular}

CIP, ciprofloxacin; LVX, levofloxacin; MXF, moxifloxacin; ND, not determined; OFX, ofloxacin.

${ }^{a}$ Codon in which heterogeneity was reported.

${ }^{\text {b}}$ Studies in which MIC was measured for levofloxacin and/or ciprofloxacin. In Takiff et al., ${ }^{3}$ Bozeman et al., ${ }^{61}$ Xu et al. ${ }^{63}$ and Soudani et al., ${ }^{11}$ MIC was measured only for ciprofloxacin; in Perlman et al. ${ }^{60}$ and Yin and Yu, ${ }^{43}$ MIC was measured only for levofloxacin.

'Studies in which MIC is not specified for every distinct mutation.

Table 7. GyrA and GyrB substitutions demonstrated and not demonstrated to confer fluoroquinolone resistance in M. tuberculosis

\begin{tabular}{|c|c|c|c|c|}
\hline \multicolumn{2}{|c|}{ Gyrase subunit alteration } & \multicolumn{2}{|c|}{$\mathrm{IC}_{50}(\mathrm{mg} / \mathrm{L})$} & \multirow[b]{2}{*}{ Study reference } \\
\hline GyrA & GyrB & OFX & MXF & \\
\hline WT & WT & $2-10$ & $1-2$ & 18,37 \\
\hline Thr80Ala & WT & 5 & 1 & 18 \\
\hline Ala90Gly & WT & 10 & 2 & 18 \\
\hline Thr80Ala+Ala90Gly & WT & 2.5 & 0.5 & 18 \\
\hline Glu21Gln+Ser95Thr+Gly668Asp+Ala74Ser & WT & 16 & 14 & 37 \\
\hline Gly88Ala & WT & 40 & 10 & 64 \\
\hline Gly88Cys & WT & 50 & 35 & 64 \\
\hline Ala90Val & WT & 100 & 35 & 18 \\
\hline Asp94Gly & WT & 350 & 50 & 18 \\
\hline Asp94His & WT & 800 & 90 & 18 \\
\hline Ala90Val + Asp94Gly & WT & $>1600$ & $>160$ & 18 \\
\hline WT & Asn499Asp & 120 & 35 & 18 \\
\hline
\end{tabular}

IC $_{50}, 50 \%$ inhibitory concentration (measured by inhibition of 50\% of DNA supercoiling); MXF, moxifloxacin; OFX, ofloxacin; WT, wild-type.

Substitutions not demonstrated to confer fluoroquinolone resistance in $M$. tuberculosis are in italics.

Substitutions demonstrated to confer fluoroquinolone resistance in M. tuberculosis are in bold.

There were some limitations of this review. First, research studies published in languages other than English were not included. Given that some mutations have been reported more frequently in certain populations, we may have excluded mutations associated with fluoroquinolone resistance simply by excluding non-English studies. Second, genotyping lineage information was only available for 10 of the 42 studies reviewed; this information would have been useful in differentiating the polymorphisms predominant in a specific lineage. Third, MEDLINE was the only database searched. Although it contains more than 20 million citations, other databases may contain publications that would have met the inclusion criteria for this study.

This extensive review of mutations described in the gyrA and gyrB genes in conjunction with the proposed consensus numbering system will facilitate the identification of novel mutations in M. tuberculosis isolates. New molecular testing methods may benefit from this information to enhance detection of fluoroquinolone-resistant M. tuberculosis.

\section{Acknowledgements}

We thank Wladimir Sougakoff and Stéphanie Petrella for helpful discussions regarding sequence alignments and corresponding numbering systems between E. coli and M. tuberculosis GyrA and GyrB. We also thank Alix Pantel for her help in searching studies reporting gyrA and gyrB mutations.

\section{Funding}

This work was supported by the National Institute of Allergy and Infectious Diseases (NIAID) (R01 AI 063200, K24 AI 65298, T32 AI 07474) and European Society of Clinical Microbiology and Infectious Diseases (ESCMID) research grant 2010.

\section{Transparency declarations}

Conflicts of interest: none to declare. 
The study sponsors did not contribute to the design, conduct, interpretation or writing of the article for this project.

\section{Author contributions}

F. M., A. W. K. and A. A. performed the literature review. F. M. wrote the first draft of the article. T. R. S. designed the study and provided substantial input in interpreting the data and writing the article. A. B., Y. F. van der $\mathrm{H}$. and C. M. assisted with the literature review and cross-referencing of information. E. C. and A. A. provided substantial input in interpreting the data and writing the article.

\section{Supplementary data}

Figures S1, S2 and S3 are available as Supplementary data at JAC Online (http://jac.oxfordjournals.org/).

\section{References}

1 American Thoracic Society, Centers for Disease Control and Prevention, and Infectious Diseases Society of America. Treatment of tuberculosis. MMWR Recomm Rep 2003; 52: 1-77.

2 Cole ST, Brosch R, Parkhill J et al. Deciphering the biology of Mycobacterium tuberculosis from the complete genome sequence. Nature 1998; 393: 537-44.

3 Takiff HE, Salazar L, Guerrero C et al. Cloning and nucleotide sequence of Mycobacterium tuberculosis gyrA and gyrB genes and detection of quinolone resistance mutations. Antimicrob Agents Chemother 1994; 38: $773-80$.

4 Mdluli K, Ma Z. Mycobacterium tuberculosis DNA gyrase as a target for drug discovery. Infect Disord Drug Targets 2007; 7: 159-68.

5 Hooper D, Rubinstein E. Quinolone Antimicrobial Agents. Washington, DC: ASM Press, 2008.

6 Cheng AF, Yew WW, Chan EW et al. Multiplex PCR amplimer conformation analysis for rapid detection of gyrA mutations in fluoroquinolone-resistant Mycobacterium tuberculosis clinical isolates. Antimicrob Agents Chemother 2004; 48: 596-601.

7 Lee AS, Tang LL, Lim IH et al. Characterization of pyrazinamide and ofloxacin resistance among drug resistant Mycobacterium tuberculosis isolates from Singapore. Int J Infect Dis 2002; 6: 48-51.

8 Pitaksajjakul P, Wongwit W, Punprasit W et al. Mutations in the gyrA and gyrB genes of fluoroquinolone-resistant Mycobacterium tuberculosis from TB patients in Thailand. Southeast Asian J Trop Med Public Health 2005; 36 Suppl 4: 228-37.

9 Huang TS, Kunin CM, Shin-Jung Lee $S$ et al. Trends in fluoroquinolone resistance of Mycobacterium tuberculosis complex in a Taiwanese medical centre: 1995-2003. J Antimicrob Chemother 2005; 56: 1058-62.

10 Siddiqi N, Shamim M, Hussain S et al. Molecular characterization of multidrug-resistant isolates of Mycobacterium tuberculosis from patients in North India. Antimicrob Agents Chemother 2002; 46: 443-50.

11 Soudani A, Hadjfredj S, Zribi M et al. First report of molecular characterization of fluoroquinolone-resistant Mycobacterium tuberculosis isolates from a Tunisian hospital. Clin Microbiol Infect 2010; 16: $1454-7$.

12 Sullivan EA, Kreiswirth BN, Palumbo $L$ et al. Emergence of fluoroquinolone-resistant tuberculosis in New York City. Lancet 1995; 345: $1148-50$.

13 Hegde SS, Vetting MW, Roderick SL et al. A fluoroquinolone resistance protein from Mycobacterium tuberculosis that mimics DNA. Science 2005; 308: $1480-3$.
14 Pasca MR, Guglierame P, Arcesi F et al. Rv2686c-Rv2687c-Rv2688c, an $A B C$ fluoroquinolone efflux pump in Mycobacterium tuberculosis. Antimicrob Agents Chemother 2004; 48: 3175-8.

15 Takiff HE, Cimino M, Musso MC et al. Efflux pump of the proton antiporter family confers low-level fluoroquinolone resistance in Mycobacterium smegmatis. Proc Natl Acad Sci USA 1996; 93: 362-6.

16 Feuerriegel S, Cox HS, Zarkua $N$ et al. Sequence analyses of just four genes to detect extensively drug-resistant Mycobacterium tuberculosis strains in multidrug-resistant tuberculosis patients undergoing treatment. Antimicrob Agents Chemother 2009; 53: 3353-6.

17 Antonova OV, Gryadunov DA, Lapa SA et al. Detection of mutations in Mycobacterium tuberculosis genome determining resistance to fluoroquinolones by hybridization on biological microchips. Bull Exp Biol Med 2008; 145: 108-13.

18 Aubry A, Veziris N, Cambau E et al. Novel gyrase mutations in quinolone-resistant and -hypersusceptible clinical isolates of Mycobacterium tuberculosis: functional analysis of mutant enzymes. Antimicrob Agents Chemother 2006; 50: 104-12.

19 Hillemann D, Rusch-Gerdes S, Richter E. Feasibility of the GenoType MTBDRsl assay for fluoroquinolone, amikacin-capreomycin, and ethambutol resistance testing of Mycobacterium tuberculosis strains and clinical specimens. J Clin Microbiol 2009; 47: 1767-72.

20 Kam KM, Yip CW, Cheung TL et al. Stepwise decrease in moxifloxacin susceptibility amongst clinical isolates of multidrug-resistant Mycobacterium tuberculosis: correlation with ofloxacin susceptibility. Microb Drug Resist 2006; 12: 7-11.

21 Mokrousov I, Otten T, Manicheva O et al. Molecular characterization of ofloxacin-resistant Mycobacterium tuberculosis strains from Russia. Antimicrob Agents Chemother 2008; 52: 2937-9.

22 Perdigao J, Macedo R, Malaquias A et al. Genetic analysis of extensively drug-resistant Mycobacterium tuberculosis strains in Lisbon, Portugal. J Antimicrob Chemother 2010; 65: 224-7.

23 Sekiguchi J, Miyoshi-Akiyama T, Augustynowicz-Kopec E et al. Detection of multidrug resistance in Mycobacterium tuberculosis. J Clin Microbiol 2007; 45: 179-92.

24 Sulochana S, Narayanan S, Paramasivan CN et al. Analysis of fluoroquinolone resistance in clinical isolates of Mycobacterium tuberculosis from India. J Chemother 2007; 19: 166-71.

25 Umubyeyi AN, Rigouts L, Shamputa IC et al. Limited fluoroquinolone resistance among Mycobacterium tuberculosis isolates from Rwanda: results of a national survey. J Antimicrob Chemother 2007; 59: 1031-3.

26 Von Groll A, Martin A, Jureen P et al. Fluoroquinolone resistance in Mycobacterium tuberculosis and mutations in gyrA and gyrB. Antimicrob Agents Chemother 2009; 53: 4498-500.

27 Williams KJ, Chan R, Piddock LJ. gyrA of ofloxacin-resistant clinical isolates of Mycobacterium tuberculosis from Hong Kong. J Antimicrob Chemother 1996; 37: 1032-4.

28 Chang KC, Yew WW, Chan RC. Rapid assays for fluoroquinolone resistance in Mycobacterium tuberculosis: a systematic review and meta-analysis. J Antimicrob Chemother 2010; 65: 1551-61.

29 Kim SJ, Espinal MA, Abe C et al. Is second-line anti-tuberculosis drug susceptibility testing reliable? Int J Tuberc Lung Dis 2004; 8: 1157-8.

30 WHO. Multidrug and Extensively Drug-Resistant TB (M/XDR-TB): 2010 Global Report on Surveillance and Response. Geneva: WHO, 2010. http://whqlibdoc.who.int/publications/2010/9789241599191_eng.pdf (13 June 2011, date last accessed).

31 Giannoni F, Iona E, Sementilli $F$ et al. Evaluation of a new line probe assay for rapid identification of gyrA mutations in Mycobacterium tuberculosis. Antimicrob Agents Chemother 2005; 49: 2928-33. 
32 Kiet VS, Lan NT, An DD et al. Evaluation of the MTBDRsl test for detection of second-line-drug resistance in Mycobacterium tuberculosis. J Clin Microbiol 2010; 48: 2934-9.

33 Brossier F, Veziris N, Aubry A et al. Detection by GenoType MTBDRs test of complex mechanisms of resistance to second-line drugs and ethambutol in multidrug-resistant Mycobacterium tuberculosis complex isolates. J Clin Microbiol 2010; 48: 1683-9.

34 Camus JC, Pryor MJ, Medigue C et al. Re-annotation of the genome sequence of Mycobacterium tuberculosis H37Rv. Microbiology 2002; 148: $2967-73$.

35 Sandgren A, Strong M, Muthukrishnan $P$ et al. Tuberculosis drug resistance mutation database. PLoS Med 2009; 6: e2.

36 Kocagoz T, Hackbarth CJ, Unsal I et al. Gyrase mutations in laboratoryselected, fluoroquinolone-resistant mutants of Mycobacterium tuberculosis H37Ra. Antimicrob Agents Chemother 1996; 40: 1768- 74.

37 Lau RW, Ho PL, Kao RY et al. Molecular characterization of fluoroquinolone resistance in Mycobacterium tuberculosis: functional analysis of gyrA mutation at position 74. Antimicrob Agents Chemother 2010; 55: 608-14.

38 Alangaden GJ, Manavathu EK, Vakulenko SB et al. Characterization of fluoroquinolone-resistant mutant strains of Mycobacterium tuberculosis selected in the laboratory and isolated from patients. Antimicrob Agents Chemother 1995; 39: 1700-3.

39 Cambau E, Sougakoff W, Besson M et al. Selection of a gyrA mutant of Mycobacterium tuberculosis resistant to fluoroquinolones during treatment with ofloxacin. J Infect Dis 1994; 170: 479-83.

40 Mitarai S. Mycobacterium tuberculosis and gyrA variation in Zambia. Trop Med Health 2005; 33: 91-4.

41 Sougakoff W, Lemaitre N, Cambau E et al. Nonradioactive single-strand conformation polymorphism analysis for detection of fluoroquinolone resistance in mycobacteria. Eur J Clin Microbiol Infect Dis 1997; 16: 395-8.

42 Duong DA, Nguyen TH, Nguyen TN et al. Beijing genotype of Mycobacterium tuberculosis is significantly associated with high-level fluoroquinolone resistance in Vietnam. Antimicrob Agents Chemother 2009; 53: 4835-9.

43 Yin X, Yu Z. Mutation characterization of gyrA and gyrB genes in levofloxacin-resistant Mycobacterium tuberculosis clinical isolates from Guangdong Province in China. J Infect 2010; 61: 150-4.

44 Zhou J, Dong Y, Zhao X et al. Selection of antibiotic-resistant bacterial mutants: allelic diversity among fluoroquinolone-resistant mutations. J Infect Dis 2000; 182: 517-25.

45 Veziris N, Martin C, Brossier F et al. Treatment failure in a case of extensively drug-resistant tuberculosis associated with selection of a GyrB mutant causing fluoroquinolone resistance. Eur J Clin Microbiol Infect Dis 2007; 26: 423-5.

46 Shi R, Zhang J, Li C et al. Emergence of ofloxacin resistance in Mycobacterium tuberculosis clinical isolates from China as determined by gyrA mutation analysis using denaturing high-pressure liquid chromatography and DNA sequencing. J Clin Microbiol 2006; 44: 4566-8.

47 Sun Z, Zhang J, Zhang $X$ et al. Comparison of gyrA gene mutations between laboratory-selected ofloxacin-resistant Mycobacterium tuberculosis strains and clinical isolates. Int J Antimicrob Agents 2008; 31: $115-21$.

48 van Doorn HR, An DD, de Jong MD et al. Fluoroquinolone resistance detection in Mycobacterium tuberculosis with locked nucleic acid probe real-time PCR. Int J Tuberc Lung Dis 2008; 12: 736-42.

49 Rinder $\mathrm{H}$, Mieskes KT, Loscher T. Heteroresistance in Mycobacterium tuberculosis. Int J Tuberc Lung Dis 2001; 5: 339-45.
50 Hofmann-Thiel S, van Ingen J, Feldmann $\mathrm{K}$ et al. Mechanisms of heteroresistance to isoniazid and rifampin of Mycobacterium tuberculosis in Tashkent, Uzbekistan. Eur Respir J 2009; 33: 368-74.

51 Lau RW, Ho PL, Kao RY et al. Rapid diagnosis of multidrug-resistant smear-positive pulmonary tuberculosis. Int J Antimicrob Agents 2010; 35: $202-3$

52 Zhang Y, Yew WW. Mechanisms of drug resistance in Mycobacterium tuberculosis. Int J Tuberc Lung Dis 2009; 13: 1320-30.

53 Evans J, Segal H. Novel multiplex allele-specific PCR assays for the detection of resistance to second-line drugs in Mycobacterium tuberculosis. J Antimicrob Chemother 2010; 65: 897-900.

54 Chan RC, Hui M, Chan EW et al. Genetic and phenotypic characterization of drug-resistant Mycobacterium tuberculosis isolates in Hong Kong. J Antimicrob Chemother 2007; 59: 866-73.

55 Bravo LT, Tuohy MJ, Ang C et al. Pyrosequencing for rapid detection of Mycobacterium tuberculosis resistance to rifampin, isoniazid, and fluoroquinolones. J Clin Microbiol 2009; 47: 3985-90.

56 Post FA, Willcox PA, Mathema B et al. Genetic polymorphism in Mycobacterium tuberculosis isolates from patients with chronic multidrug-resistant tuberculosis. J Infect Dis 2004; 190: 99-106.

57 WHO. Guidelines for the Programmatic Management of Drug-resistant Tuberculosis. Geneva: WHO, 2008. http://apps.who.int/bookorders/ anglais/detart1.jsp?sesslan=\&codlan $=\& \operatorname{codcol}=15 \& \operatorname{codcch}=2663$ June 2011, date last accessed).

58 Poissy J, Aubry A, Fernandez C et al. Should moxifloxacin be used for the treatment of extensively drug-resistant tuberculosis? An answer from a murine model. Antimicrob Agents Chemother 2010; 54: 4765-71.

59 Feasey NA, Pond M, Coleman D et al. Moxifloxacin and pyrazinamide susceptibility testing in a complex case of multidrug-resistant tuberculosis. Int J Tuberc Lung Dis 2011; 15: 417-20.

60 Perlman DC, El Sadr WM, Heifets LB et al. Susceptibility to levofloxacin of Mycobacterium tuberculosis isolates from patients with HIV-related tuberculosis and characterization of a strain with levofloxacin monoresistance. Community Programs for Clinical Research on AIDS 019 and the AIDS Clinical Trials Group 222 Protocol Team. AIDS 1997; 11: 1473-8.

61 Bozeman L, Burman W, Metchock B et al. Fluoroquinolone susceptibility among Mycobacterium tuberculosis isolates from the United States and Canada. Clin Infect Dis 2005; 40: 386-91.

62 Sun Z, Chao Y, Zhang $X$ et al. Characterization of extensively drug-resistant Mycobacterium tuberculosis clinical isolates in China. J Clin Microbiol 2008; 46: 4075-7.

$63 \mathrm{Xu}$ C, Kreiswirth BN, Sreevatsan S et al. Fluoroquinolone resistance associated with specific gyrase mutations in clinical isolates of multidrugresistant Mycobacterium tuberculosis. J Infect Dis 1996; 174: 1127-30.

64 Matrat S, Veziris N, Mayer C et al. Functional analysis of DNA gyrase mutant enzymes carrying mutations at position 88 in the A subunit found in clinical strains of Mycobacterium tuberculosis resistant to fluoroquinolones. Antimicrob Agents Chemother 2006; 50: 4170-3.

65 Siddiai N, Shamim M, Jain NK et al. Molecular genetic analysis of multi-drug resistance in Indian isolates of Mycobacterium tuberculosis. Mem Inst Oswaldo Cruz 1998; 93: 589-94.

66 Wang JY, Lee LN, Lai HC et al. Fluoroquinolone resistance in Mycobacterium tuberculosis isolates: associated genetic mutations and relationship to antimicrobial exposure. J Antimicrob Chemother 2007; 59: 860-5.

67 Pantel A, Brossier F, Bastian S. Mutations in the GyrB subunit of clinical Mycobacterium tuberculosis strains: consequences on resistance to fluoroquinolones. In: Abstracts of the Fiftieth Interscience Conference on Antimicrobial Agents and Chemotherapy, Boston, MA, 2010. Abstract C1-085. American Society for Microbiology, Washington, DC, USA. 